\title{
science \\ Role of academic publishers in 10 years: a perspective from the Chairman of Elsevier
}

\author{
Youngsuk Chi \\ Elsevier, New York, NY, USA
}

\section{Introduction}

While it is unfortunate that we are unable to come together in person, I believe that there is a silver lining in this coronavirus disease 2019 (COVID-19) pandemic for the global publishing industry. The pandemic has allowed us to pause and reflect on our recent progress so that we can accelerate into an exciting new era of scientific research and discovery.

I would like to sincerely congratulate you on the 10th anniversary of the Korean Council of Science Editors (KCSE). With its progress over the last decade, KCSE is making great strides in its mission to improve the quality of Korean scientific journals. I am confident that the recent partnership between KCSE and Elsevier on the Scopus Expert Content Selection \& Advisory Committee-Korea (ECSAC-Korea) will spur even more developments in the decades to come [1]. In the last ten years since KCSE's founding, the role of publishers has changed significantly to adapt to the evolving needs of the scientific community. So today, I would like to discuss how these roles have transformed and how these transformations will influence the future of our industry.

\section{Transformation: The Role of Netflix}

Received: December 29, 2021 Accepted: January 5, 2022

Correspondence to Youngsuk Chi ys@elsevier.com

ORCID

Youngsuk Chi

https://orcid.org/0000-0003-4729-0466

This article is based on a plenary lecture presented at the 10th Anniversary Symposium of the Korean Council of Science Editors through Zoom on September 8, 2021 (Korea standard time).
Before I dive into our discussion, I would like to talk about a company that arose from humble beginnings to redefine its roles and industry. I am sure that almost everyone has heard of Netflix, and binge-watched a couple of movies and TV shows on their platform during 2020's lockdowns.

Well, Netflix was not always the popular movie-streaming platform it is today. The company began in 1997 as a simple DVD-delivery company. It did not create anything particularly groundbreaking; it simply cataloged and distributed films. From the start, however, its founders understood the importance of data. Co-founder Reed Hastings earned a master's degree in artificial intelligence from Stanford University in 1988, talk about ahead of the curve. After their founding, Netflix launched a personalization algorithm that used member ratings to predict customers' tastes and recommend new titles. Because of this remarkable customization system, Netflix's subscriber count accelerated quickly, from 1.41 million in 2003 to 4.02 million in 2005 [2]. 
But they did not just stop there. They continued to innovate boldly and added online streaming to their subscription plans in 2007. By 2010, they had become the Netflix we know and love. Netflix expanded worldwide, and by 2016 it operated in 190 countries, including Korea. While growing, Netflix established a studio and began producing original content in many different languages, including award-winning TV shows like "Kingdom," "Mr. Sunshine," and "Hospital Playlist (슬기로운 의사생활)" (Fig. 1). To date, Netflix has made over 80 original Korean TV shows and films and plans to spend half a billion more dollars on Korean content this year alone. But even as Netflix expanded internationally, their data analysis capabilities remained the core of their business. Today, their personalization algorithm alone is worth 2 billion US dollars. But what does a movie company like Netflix have to do with publishing? Well, in just under a decade, Netflix defined a new role for itself by harnessing data analytics to change the way customers consumed and interacted with content. When Netflix switched to streaming, few of its competitors envisioned that it would be successful. But when combined with a deep understanding of data, their streaming model became the gold standard for media. Now, everyone follows Netflix's lead, leaving the entire media and entertainment industry looking dramatically different than it did before.

\section{The Three New Roles of Publishers}

Much like Netflix, publishers have evolved into new roles centered around technology and development in the last decade, and in doing so, transformed the science, technology, and medicine (STM) industry. Today's publishers do much more than just peer review, format, print, package, and sell authors' work. Instead, we explore new publishing models to find better ways to meet customer needs and influence the trends that define STM. These trends include the new roles of publishers in social responsibility, research solutions and technology, and Open Science (Fig. 2). Together, they are the foundation upon which we will build the next decade of publishing!

\section{Social responsibility for sustainable development and gender and racial diversity}

The first trend I would like to discuss is how new roles in research have brought on more responsibility for publishers in society. For the modern publisher, social responsibility is interwoven into the company's DNA. The last decade has demonstrated the importance of publishers as leaders in social issues, such as data ethics and the Inclusion and Diversity in research. Luckily, as the world grows more aware of these challenges, we as publishers have taken on new roles in ensuring that our content and practices are ethical.

Take the UN Sustainable Development Goals (SDGs), for example. Last year, dozens of publishers signed the SDG Publisher's Compact, committing to 10 action points to accelerate progress on the 17 goals. These publishers are combining their unique insights with expertise from the research community to drive innovation. Already, some publishers have taken concrete steps to achieve these goals, too. At Elsevier, we renewed our commitment to the SDGs in July by signing the Climate Pledge. In addition to internal changes like reducing business travel, we have created new journals focused on climate action, such as One Earth from Cell Press and Lancet Planetary Health (Fig. 3). We even mapped each of our journals' progress towards the SDGs they support. These actions show that publishers use their unique insights to lead concerted efforts
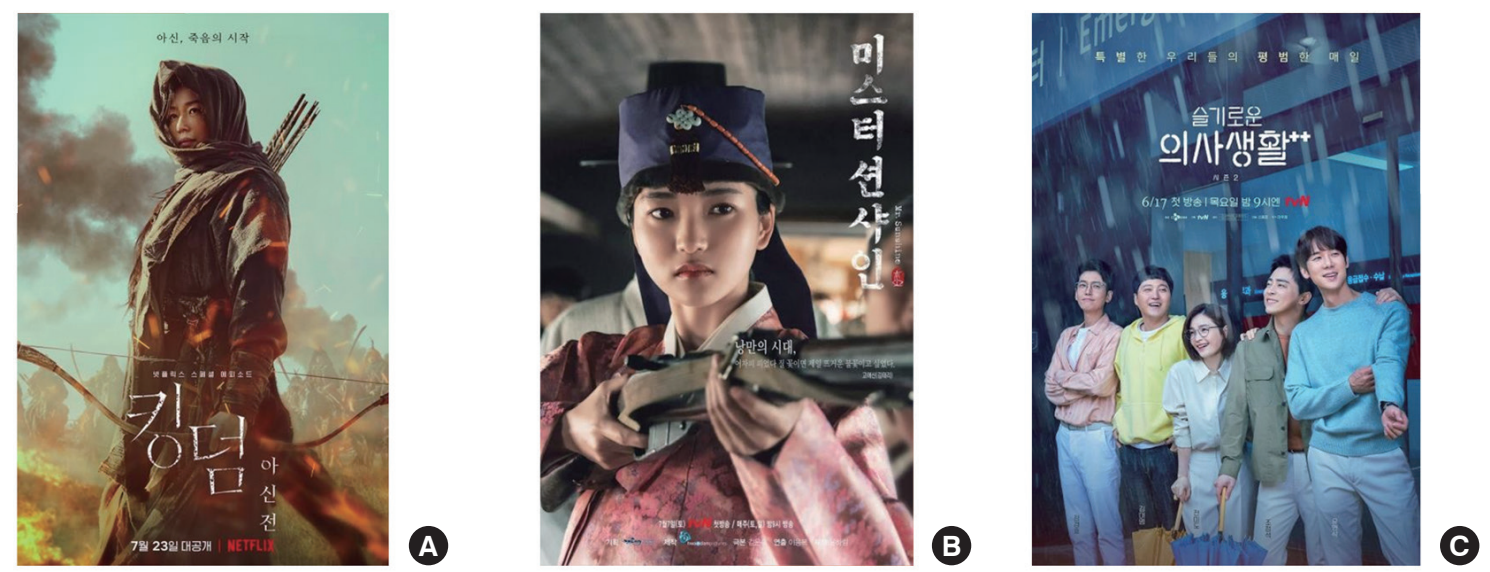

Fig. 1. TV shows (A) "Kingdom," (B) "Mr. Sunshine," and (C) "Hospital Playlist (슬기로운 의사생활)" produced in Korea and provided through the Netflix. Sources: (A) https://www.koreatimes.co.kr/www/art/2021/07/688_312472.html, (B) https://www.imdb.com/title/tt7094780/mediaviewer/rm1700377857/, and (C) https://www.imdb.com/title/tt11769304/. 


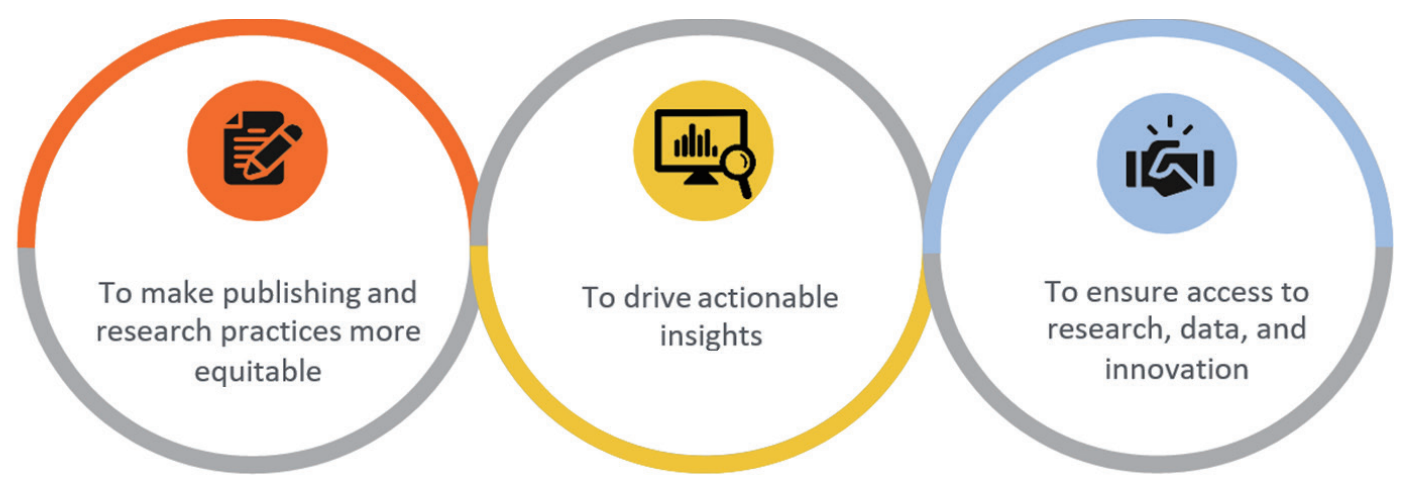

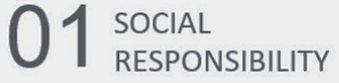
02 Rolurions
03 SOAEN

Fig. 2. Diagram of changing roles in publishing, including social responsibility, research solutions, and open science. Produced by the author.
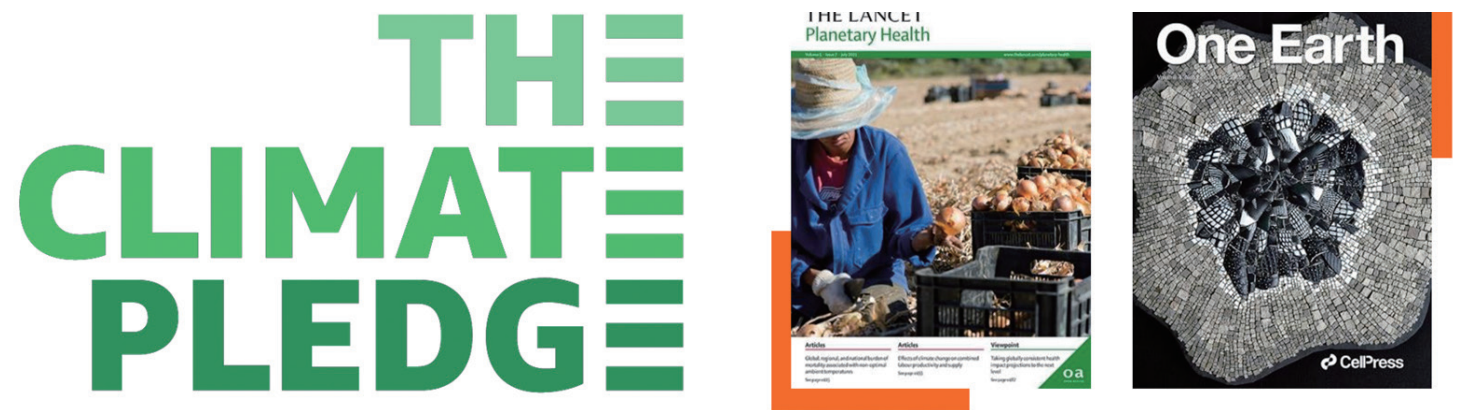

Fig. 3. Elsevier's two new journals on the climate pledge and the UN Sustainable Development Goals, One Earth from Cell Press and Lancet Planetary Health.

to combat our world's biggest problems. This new role in social responsibility also includes an examination of Inclusion and Diversity, both within the publishing industry and academic research fields.

Diversity in research is especially crucial, and excluding researchers can have dire real-life consequences. For example, a study in Britain found that women were 50\% more likely to be misdiagnosed following a heart attack, largely because most clinical trials use male participants.

Publishers have taken important steps to improve gender and racial diversity in response to these issues. Elsevier's gender reports benchmark progress and identify systemic barriers to equality. All our journals have published gender goals measured by discipline benchmarks. We also use our advanced data capabilities to combat gender inequality through programs like SHE figures and MINDtheGEPs (Modifying Institutions by Developing Gender Equality Plans) (Fig. 4).

A global commitment to social responsibility will fuel the next great innovations and breakthroughs in our industry and the entire scientific community. And while we have made a lot of progress, there is still a long way to go. As UN SecretaryGeneral Antonio Guterres warned in 2019, no country is on track to meet the SDG goal of gender equality [3]. His prediction is especially relevant in Korea. While women in science, technology, engineering, and mathematics research \& development increased significantly over the last decade, 181,972 out of 718,759 (25.3\%) of R\&D personnel in Korea were women in 2019 [4]. Luckily, one of the beauties of the publishing world is that we can amplify other voices and bring important regional or social issues into the global spotlight. So, let's keep striving towards this new and exciting role in the next decade.

Solutions in publishing technologies built on AI, machine learning, and data

Our second trend, the growing role of technology in publishing solutions, builds on the foundation of ethics and social responsibility. The last decade of STM publishing has seen unprecedented data-driven insights and research workflows. Technologies built on AI, machine learning, and data will be used to increase publication and make publishing better. 


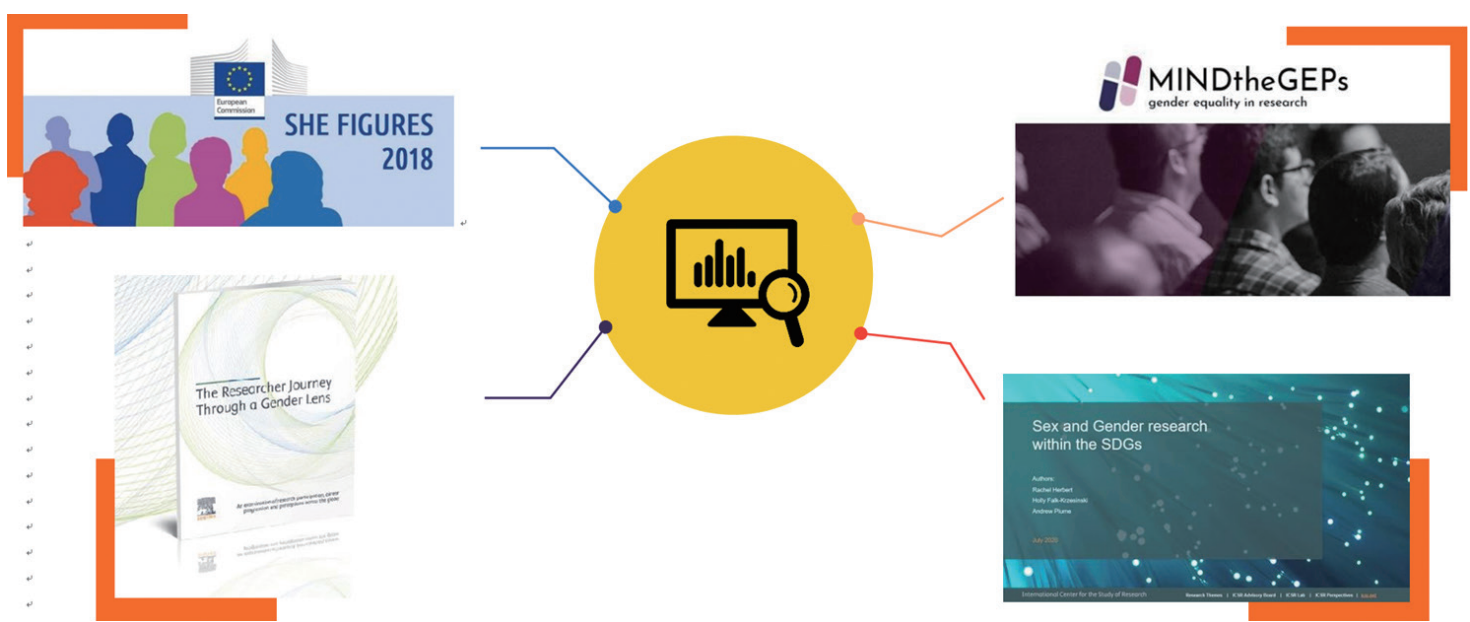

Fig. 4. Strategies or activities of combatting the gender inequality by publishers and organizations, including SHE GIFURES 2018, the Research Journey Through a Gender Lens, MINDtheGEPs (Modifying Institutions by Developing Gender Equality Plans), and Sex and Gender Research with the SDGs (Sustainable Development Goals).

Let me share with you an analogy from Elsevier to explain what I mean. When Elsevier first started in the print environment, we were like farmers. We would produce journals and "harvest" information without knowing how our products were used. In the 1990s, at the start of the internet era, Elsevier evolved into a supermarket. When we adopted electronic distribution, we consolidated everything we had farmed into one place, where library and researcher customers could pick out exactly what they needed. But the key to our success in the last decade is that we did not just stop at electronic content.

Now, we have become a restaurant serving artificial intelligence and machine learning-powered solutions. We work directly with our customers to create "recipes" catered to their unique wants and needs, using artificial intelligence and machine learnig as our key ingredients. The content volume is only increasing in today's market, making our analytical insights even more important for streamlining the research process.

At Elsevier, the focus on workflows has taken several different paths. We have tools like Mendeley (https://www.mendeley. $\mathrm{com} /$ ) that help connect researchers with relevant papers based on what they are researching. Our Pure tool (https:// www.elsevier.com/solutions/pure) works with researchers to improve decision-making, data, and functionality in all project areas. And we have also developed an 'Article Recommender' [5] to help researchers connect with the essential information they might not have otherwise discovered. Offering analytical tools like these demonstrates the evolution of publishers' roles, from research facilitators to initiators of insights. And our new role in technology is only made possible by our commitment to ethics and social responsibility, which helps us solve the problems that arise with new technologies.

The emergence of data-centered technology and widespread information sharing has spurred greater oversight of responsible companies. Every industry has had to deal with new issues relating to technology. Netflix, for example, had to grapple with the privacy implications of storing massive user data needed for their algorithm. The STM industry has also had to deal with new challenges arising from advancing technology. Dishonest publications can use artificial intelligence and machine learning for citation hacking and article generation, often evident through "tortured phrases." These trends could threaten the credibility of research and the journals that publish them.

Ethical publishing, then, means changing both how research is verified and how it is measured. Publishers that already had ethics in their DNA have thrived. Elsevier has invested in software and tools to uphold ethical publication standards and combat fraud. Furthermore, our International Center for the Study of Research (https://www.elsevier.com/icsr) develops new methods to advance research evaluation. Its outcomes support the responsible use of metrics and help develop best practices in research assessment and research impact. Just as Netflix turned to data analytics to enhance and customize their content, the future growth of publishers relies on the new ways to apply artificial intelligence solutions and technological tools to research workflows and publishing processes.

\section{Open Science}

The last major change in the last decade has been our new role in Open Science, a broad term for publicly accessible re- 

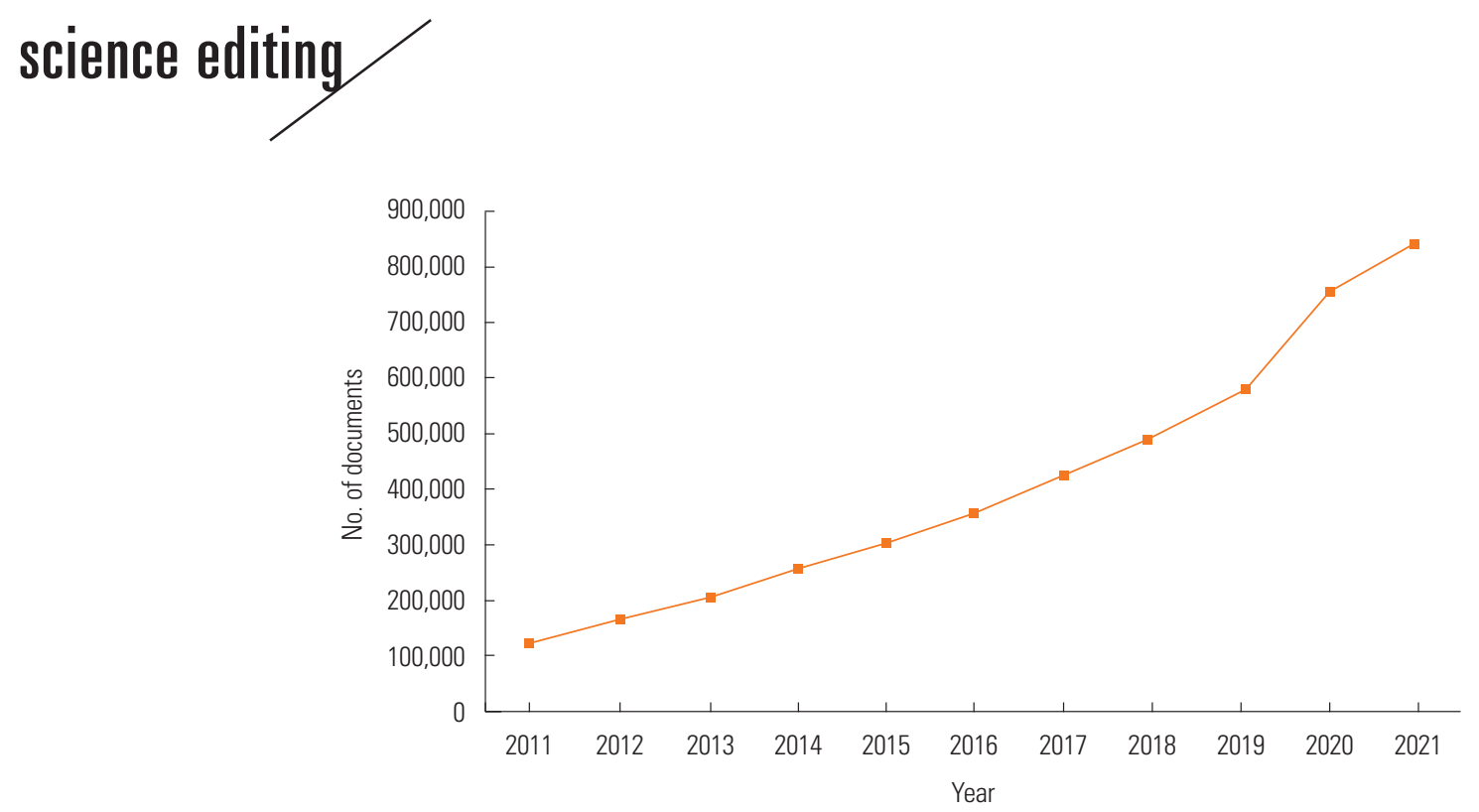

Fig. 5. Number of gold open access documents in the Scopus database according to year [cited 2021 Sep 8] (Suppl. 1).

search findings and outputs. Open Science is one way to ensure equitable access to research, data, and innovation. Open Science presents innovations in publishing, just as Netflix's online streaming services did for media and entertainment. Similar to how Netflix adopted a new way of delivering content to its customers, publishers and researchers alike are turning to diverse publishing methods for research access. Open Science is an even more complex innovation than Netflix since Open Science research published in one country benefits the global information economy.

Open access, one of the many branches of Open Science, has become increasingly popular in the last decade. As publishers, we have embraced open access, highlighted by transformative agreements. Even though we do not favor any particular publishing model, we at Elsevier are committed to enabling researchers to meet their goals, including their open access objectives. We believe that every researcher has a fundamental right to validate their work through peer review and publish it in a way that works for them. We are one of the fastest-growing open access publishers. Just look at the data: last year, we published 84,000 gold open access articles, up from just under 12,000 in 2011 (Fig. 5) (Suppl. 1). Elsevier alone has 17 commercial agreements, and over $20 \%$ of our journals are now fully gold open access-all the others allow open access publications when desired.

Elsevier is committed to working closely with Korean journals to extend Open Science opportunities. After a decade of rapid growth, Korea is already a world leader in open access. In the Scopus database, open access documents from Korea were published by $24.3 \%$ in 2011 and $47.8 \%$ in 2021 (Fig. 6) (Suppl. 2). We signed a Transformative Agreement with the National Research Council of Science and Technology of Korea in 2019 to help support these trends. With collaborations like these, Korea will continue its rapid progress in open access and Open Science. However, in the same way that movies are just one part of Netflix's diverse offering, Open access is just one small slice of the greater Open Science pie (Fig. 7).

In addition to open access, Open Science also includes open data, open metrics, open software and hardware, open protocols, and many more. Open Science aims to support trust and reproducibility in research outcomes and encourage the reuse of data for a more comprehensive analysis. So as you can see, Open Science will be a global, impactful, and unprecedented movement that truly maximizes research discoverability, transparency, and collaboration.

At Elsevier, Open Science forms part of our commitment to ensuring credible, high-quality research for our trusted partners. For example, we are helping develop research in low-income countries through Research4Life (https://www. research4life.org/) by providing the content and publishing solutions they need. This devotion is all part of our commitment to make the most significant difference in a sustainable way. We even offer free access to relevant research for health emergencies, most notably during the COVID-19 pandemic. Our Novel Coronavirus Information Center (https://www. elsevier.com/connect/coronavirus-information-center) harnessed the power of Open Science to help researchers make the timely breakthroughs that have helped fight this pandemic.

While the relationships between researchers and publishers are becoming more diverse, the fundamental support that publishers provide institutions and researchers has not changed. All parts of Open Science articles are evaluated along with the same measures of robustness, impact, novelty, and integrity as every article we publish. We never compromise on quality, but in embracing Open Science, we are building a more collaborative, inclusive, and transparent world of research. Going for- 


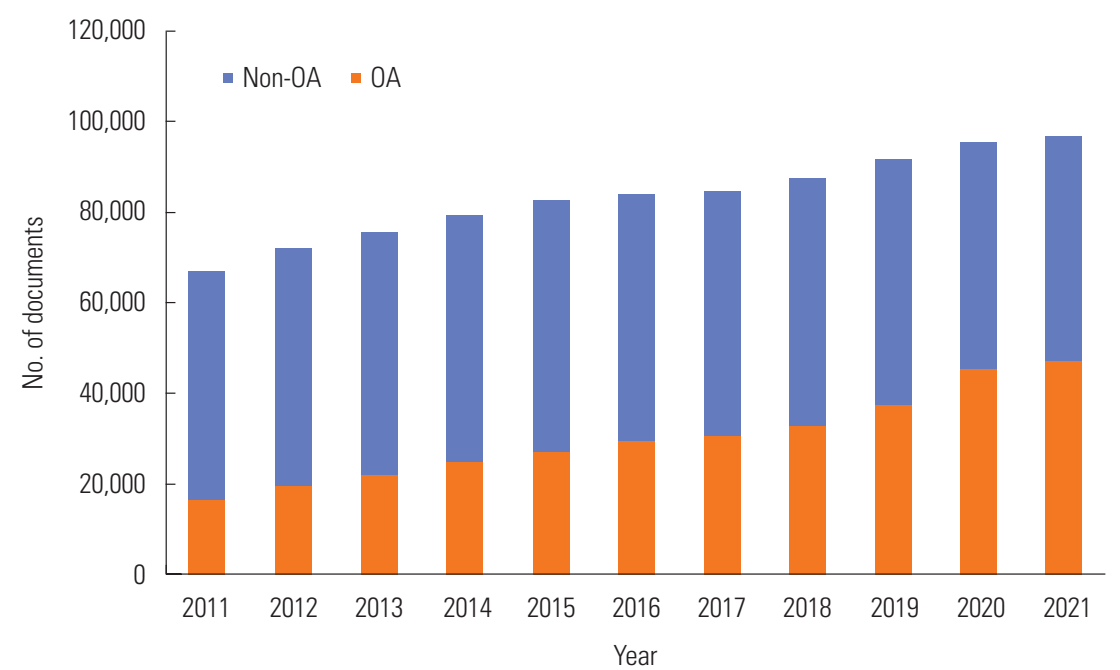

Fig. 6. Number of open access documents and non-open access documents from South Korea in the Scopus database according to year [cited 2021 Sep 8] (Suppl. 2).

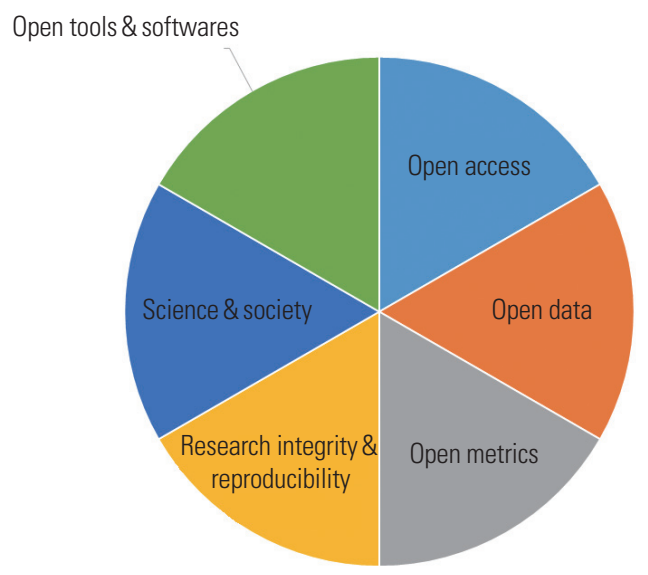

Fig. 7. Open science pie including open access, open data, open metrics, research integrity and reproducibility, science and society, and open tools and softwares.

ward, authors, researchers, and their institutions will be able to share knowledge and build on each other's work faster and more easily than ever before. Just as Netflix reinvented itself first as a revolutionary online streaming platform, then as a world leader in original storytelling, the publishing industry continues to evolve. We have made great strides leading the world in social responsibility, enhancing research through technological advancements, and pioneering new models of Open Science.

\section{Conclusion}

These trends have shaped the last decade of publishing, motivating us all to create the high-quality work that drives progress. But these three trends will also be the building blocks of innovation in the decades to come. I firmly believe that KCSE will continue its great progress in raising the international prestige of Korean journals. Elsevier is committed to supporting South Korean researchers as a valued partner for ECSACKorea. Continued international collaboration like this will increase the profile of Korea's journals while ensuring they maintain the utmost quality. So, let's continue to work together to build on the advancements of the last decade so that we will have even more to celebrate at KCSE's 20th anniversary.

\section{Conflict of Interest}

Youngsuk Chi has been the chairman of Elsevier since December 2009. He has no financial interest with Netflix mentioned as an innovative company. No other potential conflict of interest relevant to this article was reported.

\section{Funding}

The author received no financial support for this article.

\section{Supplementary Material}

Supplementary files are available from the Harvard Dataverse at: https://doi.org/10.7910/DVN/SK8K5B

Suppl. 1. Number of gold open access documents in the Scopus database according to year

Suppl. 2. Number of open access documents and non-open access documents from South Korea in the Scopus database according to year

\section{References}

1. Kim H. History of the Scopus Expert Content Selection and Advisory Committee of Korea. Sci Ed 2020;7:6-10. https:// 
doi.org/10.6087/kcse.183

2. Dean B. Netflix subscriber and growth statistics: how many people watch Netflix in 2022? [Internet]. [place unknown]: Backlinco; 2021 [cited 2021 Sep 8]. Available from: https:// backlinko.com/netflix-users

3. Guterres A. Remarks to high-level political forum on sustainable development [Internet]. New York, NY: United Nations; 2019 [cited $2021 \mathrm{Sep} 8$ 8]. Available from: https://www. un.org/sg/en/content/sg/speeches/2019-09-24/remarkshigh-level-political-sustainable-development-forum
4. Organization for Economic Cooperation and Development. R\&D personnel by sector and function [Internet]. Paris: OECD.Stat; 2021 [cited 2021 Sep 8]. Available from: https://stats.oecd.org/Index.aspx?DataSetCode=PERS_ FUNC

5. Haak W. ScienceDirect's Article Recommender gets smarter and casts a wider net [Internet]. New York, NY: Elsevier; 2014 [cited 2021 Sep 8]. Available from: https://www.elsevier. $\mathrm{com} /$ connect/archive/sciencedirects-article-recommendergets-smarter-and-casts-a-wider-net 